\title{
A newborn requiring selective bronchial intubation
}

\begin{abstract}
A preterm infant born at 27 - Aweeks' gestation had stable $\stackrel{\partial}{\circ}$ ventilatory requirements until day 3 , when a pulmonary hemहु orrhage developed.

Positive end-expiratory pressure was increased from 5 to $7 \mathrm{~mm} \mathrm{Hg}$. On day 10 a left-sided $\AA$ pneumothorax developed that required drainage with a chest tube. A chest radiograph revealed pulmonary interstitial emphysema
\end{abstract}
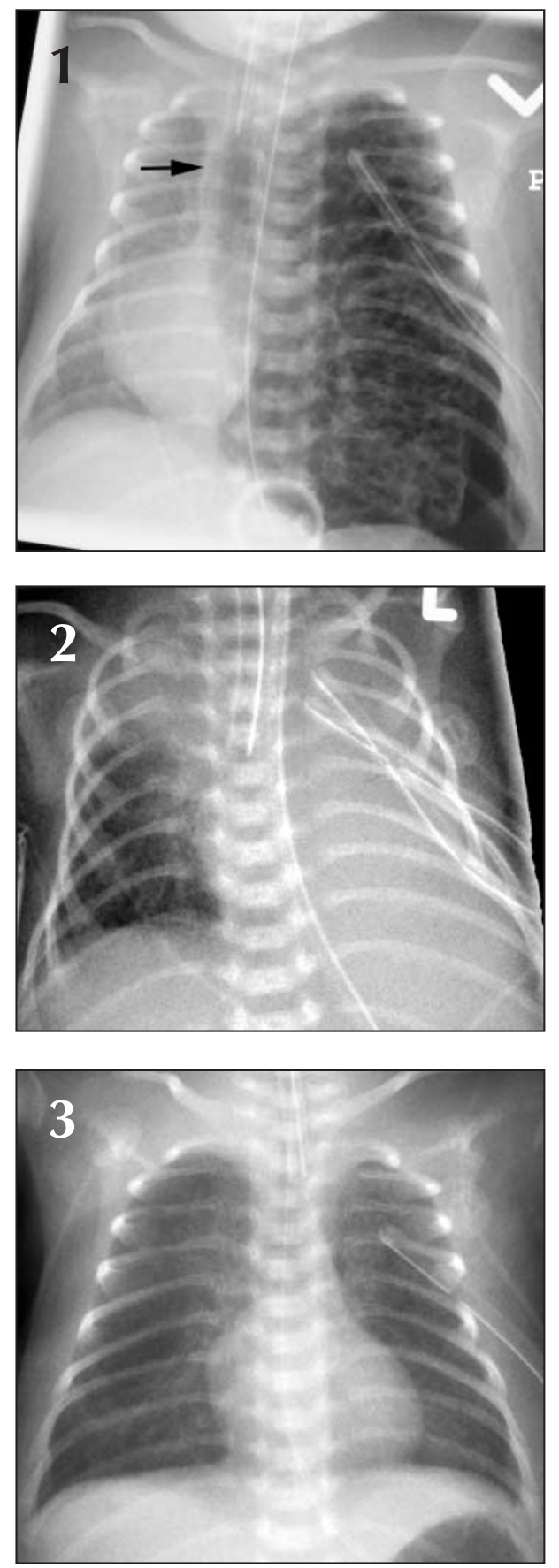

(PIE) in the left lung. There was respiratory deterioration, with worsening PIE and hyperinflation of the left lung, a residual leftsided pneumothorax and compression atelectasis of the right lung (Fig. 1; the anterior junction line is displaced [arrow] owing to herniation of the left lung anteriorly to the right). Failure of conventional therapies and worsening respiratory function prompted selective bronchial intubation for ventilation of the right lung.

The first radiograph obtained after selective intubation showed the tip of the endotracheal tube (ETT) in the right bronchus intermedius, with loss of aeration in the right upper lobe. There was almost complete loss of aeration of the left lung and no evidence of PIE (Fig. 2). The infant's clinical condition improved, and the ETT was withdrawn to mid-tracheal position after 5 days. Subsequent chest radiographs showed adequate expansion of both lungs without recurrence of PIE on the left side (Fig. 3). A few days later the tube was removed and bubble CPAP (continuous positive airway pressure) started. A CT scan of the chest at 3 weeks of age showed no sign of PIE or congenital cystic lesions.

PIE is a severe complication associated usually, although not exclusively, with mechanical ventilation in preterm neonates, ${ }^{1}$ occurring in $42 \%$ of infants with birth weights of $500-799 \mathrm{~g}, 29 \%$ of those $800-899 \mathrm{~g}$ and $20 \%$ of those 900-999 g. ${ }^{2}$ It involves collection of air in the interstitial compartment of the lung parenchyma related to lack of pores of Kohn (alveolar pores) in the immature lung, which leads to reduced collateral air movement. Adverse effects of PIE are related to decreased perfusion and ventilation of the affected lung tissue, compression of the normal adjacent lung and mediastinum, and occurrence of pneumothorax, pneumomediastinum, pneumopericardium and air embolism. The presence of PIE in very-low-birth-weight infants is associated with a high risk of death and significant long-term morbidity, including an increased risk of chronic lung disease. Although PIE has been linked to high peak inspiratory pressure, findings from animal studies suggest that it may be associated with structural and maturational factors rather than to overdistension alone.

PIE may present as diffuse bilateral involvement or a unilateral lesion. In unilateral PIE, mediastinal shift causes compressive atelectasis of the opposite lung, which leads to an increased need for higher ventilatory pressures, progressive overdistension of the affected lung and a worsening cycle of events. Selective bronchial intubation and ventilation of the other lung breaks the cycle and gives time for the interstitial emphysema to regress, with much less baro- and volutrauma. Bronchial occlusion, multiple linear thoracotomies, surgical resection and liquid ventilation have also been tried, with variable results.

\section{Minesh Khashu \\ Department of Pediatrics \\ John Mawson \\ Department of Radiology \\ Seema Bhargava \\ Avash Jeet Singh \\ Department of Pediatrics \\ Children's \& Women's Health \\ Centre of British Columbia \\ University of British Columbia \\ Vancouver, BC}

\section{References}

1. Al Jishi N, Dyer D, Sharief N, alAlaiyan S. Selective bronchial occlusion for treatment of bullous interstitial emphysema and bronchopleural fistula. $\mathcal{F}$ Pediatr Surg 1994;29(12):1545-7.

2. Yu VY, Wong PY, Bajuk B. Pulmonary interstitial emphysema in infants less than $1000 \mathrm{~g}$ at birth. Aust Paediatr F 1986;22(3): 189-92.

3. Willet KE, Jobe AH, Ikegami M, Newnham J, Sly PD. Pulmonary interstitial emphysema 24 hours after antenatal betamethasone treatment in preterm sheep. Am 7 Respir Crit Care Med 2000;162(3):1087-94. 